\title{
Influenza as an important factor causing increased risk of patients' deaths, excessive morbidity and prolonged hospital stays
}

\author{
Jarosław Drobnik ${ }^{1}$, Piotr Pobrotyn², Izabela T. Witczak³ ${ }^{3}$ Adam Antczak ${ }^{4}$, Robert Susło ${ }^{1}$
}

\author{
${ }^{1}$ Epidemiology and Health Education Division, Population Health Department, Health \\ Sciences Faculty, Wrocław Medical University, Poland \\ ${ }^{2}$ Management, University Clinical Hospital, Wroclaw, Poland \\ ${ }^{3}$ Public Health Division, Population Health Department, Health Sciences Faculty, \\ Wrocław Medical University, Poland \\ ${ }^{4}$ Department of General and Oncological Pulmonology, Medical University of Lodz, \\ Lodz, Poland
}

Submitted: 17 June 2020, Accepted: 24 May 2021

Online publication: 4 June 2021

Arch Med Sci

DOI: https://doi.org/10.5114/aoms/138145

Copyright $\odot 2021$ Termedia \& Banach

\section{A bstract}

Introduction: Influenza infection is associated with potential serious complications, increased hospitalization rates and a higher risk of death.

Material and methods: A retrospective comparative analysis of selected indicators of hospitalization at the University Hospital in Wroclaw was conducted on patients with confirmed influenza infection and a control group during the 2018-2019 influenza season. The threshold for statistical significance of differences between the groups was set at $p<0.05$.

Results: The types of influenza viruses confirmed in the hospital patients were remarkably similar to those occurring in the general population in Poland. The largest numbers of influenza cases were observed at the departments related to internal medicine where patients with cardiac, lung and renal diseases were hospitalized. The risk of death among the patients with confirmed influenza infection was significantly higher than among the other patients. The highest risk of death was observed among the patients with confirmed influenza infection at the departments related to internal medicine. Considering patients from the entire hospital, the mean length of hospital stay for those with confirmed influenza was 2.13-fold longer than for those in the control group. Comparisons of the median, minimum and maximum lengths of hospitalization between the patients with confirmed influenza infection and the control group reveal even more distinct differences. Conclusions: Significant differences in the selected indicators of hospitalization were observed between the patients with confirmed influenza infection and the control group; they are associated with serious social costs, such as prolonged hospital stay and a higher risk of death during hospitalization in Poland.

Key words: mortality, aged, morbidity, medical economics, length of stay, multimorbidity, influenza vaccines, human influenza, hospital economics.

\section{Introduction}

Influenza and influenza-like infections are seasonal issues. Influenza infections occur as an epidemic or (less frequently) a pandemic, incurring significant direct, indirect and social costs [1] as about 500,000 patients

\author{
Corresponding author: \\ Dr. Robert Susło \\ Epidemiology and Health \\ Education Division \\ Population Health \\ Department \\ Health Sciences Faculty \\ Wrocław Medical University \\ Wroclaw, Poland \\ Phone: +48717841813 \\ E-mail: robert.suslo@umw. \\ edu.pl
}


and families are impacted each year worldwide [2]. The morbidity among children reaches as high as $30 \%$, the highest number of influenza cases is reported in children under 14 years old, and the frequency of complications among hospitalized children is as high as $57 \%$, which requires prolonged hospitalizations and significantly increases their costs [3]. The social costs stem from a poorer quality of life, exclusion of influenza patients from their daily routines (including study or work), the treatment costs and from necessary care being provided to the patients, potentially and as needed, by others - mostly informal caregivers (family members in particular). According to the World Health Organization's (WHO) recommendations, confirmed and suspected influenza cases are registered by the National Influenza Centre (Krajowy Ośrodek ds. Grypy) and the Department of Influenza Research (Zakład Badania Wirusów Grypy) at the National Institute of Public Health - National Institute of Hygiene (Narodowy Instytut Zdrowia Publicznego - Państwowy Zakład Higieny [NIZP-PZH]) [4] over a period of 12 months, from 1 October to the end of September the following year. In view of all this, influenza constitutes a serious concern - from the point of view of both the government and the general public $[5,6]$.

In the case of homogeneous groups of patients any well-defined comorbidity adequately predicts mortality [7]. The numbers of influenza cases and flu-related fatalities seem to be remarkably underestimated, particularly in older age groups. Before the influenza immunization programme was instituted in the Netherlands, there were on average 2.6 influenza deaths registered as fatalities, which were caused by other factors, for every registered influenza death from 1967 to 1989; therefore, the numbers included in the formal statistics of influenza mortality should be multiplied by at least 3.6 [8]. Moreover, only $5 \%$ of influenza fatalities that were erroneously attributed to other causes referred to patients under 60 years of age, and this rate increased among older age groups: from $12 \%$ for the range of 60 to 69 years, through $29 \%$ for the 70-79 age group, up to 54\% for patients $80+$ years old. Importantly, most commonly, the influenza deaths were wrongly reported as being caused by cardiovascular (47\%) or respiratory (23\%) diseases as well as other illnesses (30\%) [8]. A similar issue, to various extents, was reported to be true in case of infectious diseases other than influenza, including the recent COVID-19 pandemic-related deaths [9]. Despite problems finding precise data, it is known that seasonal influenza epidemics significantly increase mortality and carry a risk of complications; particularly sensitive groups are children younger than one year, adults aged 65 and older, pregnant women and patients with comorbidities. Every year, there are about 35,000 influenza-related deaths and 200,000 inpatient cases reported in the USA [10], while in European countries influenza epidemics usually affect approximately $5 \%$ to $15 \%$ of the population. For the regions of Central and Eastern Europe, influenza infections require the hospitalization of approximately 30 additional patients per 100,000 inhabitants, concerning patients aged 65 and older. Among these countries, Poland bears the highest estimated additional costs - over 5 million EUR per year [11]. Early introduction of intensive medical treatment is expensive, but - like pre-emptive intensive care unit admissions of not yet ventilated patients - has a potential to reduce excessive mortality in Poland as such patients have a significantly higher potential for a favourable outcome [12]. Unfortunately, in the case of serious influenza complications, the availability of expensive advanced treatment methods is limited in Poland, which may even lead to patients dying while on the waiting list, as in the case of pulmonary fibrosis [13]. Unfortunately, the popularity of vaccine programmes is still unsatisfactory despite seasonal influenza being a serious health concern, especially for the elderly or patients with comorbidities. Not only do the elderly face the most serious influenza-related health issues and risks, but vaccine effectiveness is also at the lowest level in this population due to their poorer immune response in comparison with other age groups [14]. In the case of influenza vaccines serious post-vaccination adverse reactions are rare or a causal relation between vaccination and its potential complications cannot be established at all [15]. Therefore, the cocoon strategy based on herd immunity is even more attractive for seniors, as it decreases the risk of contact with influenza viruses by reducing - via immunization - the number of people who may transmit the virus to their family, friends and neighbours [16]. However, one obstacle here is a sceptical attitude to and avoidance of immunization, which is growing stronger every year [17]. At the same time, people in Poland strongly believe that antibiotic treatment is both sufficient and required in the vast majority of infections, and the result is both high demand for antibiotic prescriptions and common antibiotic self-medication for respiratory tract infections [18]. The issue of epidemic seasonal influenza prevention has always been relevant in the context of care and nursing homes for the elderly and chronically diseased patients [19]. While making informed decisions about seasonal influenza vaccination, patients usually require proper support from the primary care physicians [20].

Also the authors' own medical practice observations indicate that many Polish patients with in- 
fluenza signs and symptoms are reluctant to seek medical assistance in fear of financial loss due to doctor's orders to temporarily refrain from work or in the hope that the infection will resolve itself without medical intervention. Another important issue is the insufficient number of confirmatory laboratory tests being performed, particularly as regards healthcare institutions without the facilities to conduct these tests on their own. Consequently, it is safe to assume that the numbers of reports concerning influenza cases and influenza suspected cases in Poland are underestimated.

The study objectives were to determine differences in selected indicators of hospitalization in a multispecialty university hospital in Poland between patients with confirmed influenza infection and a control group (individuals without confirmed influenza), as well as their effects on the inpatient treatment costs. The specific research questions were whether influenza virus infection modifies mortality of hospitalized patients, prolongs hospitalization time or increases costs of treatment. In order to confirm the generalizability potential of the study results, the authors aimed to determine the types of influenza viruses that were identified in the patients of the University
Hospital in Wroctaw compared to those occurring in the general population in Poland.

\section{Material and methods}

In the first quarter of 2019 (during the epidemic 2018-2019 influenza season), 310 cases of suspected influenza infection that were monitored for influenza and ultimately verified as negative and 212 influenza cases in total were confirmed at all departments of the University Hospital in Wroclaw. Due to lack of compatibility of information systems in different branches of the University Hospital, the complete medical records and pharmacoeconomic data were available for review only regarding the University Hospital units located at 213 Borowska Street. Consequently, comparative analysis of hospitalization indicators was conducted for 130 patients who were treated in those units with confirmed influenza during their hospital stay and for a control group of 260 individuals without confirmed influenza infection. The control group comprised patients randomly selected among people hospitalized in the other departments where the influenza cases were identified between 1 January and 31 March 2019. The size of the control group in each department

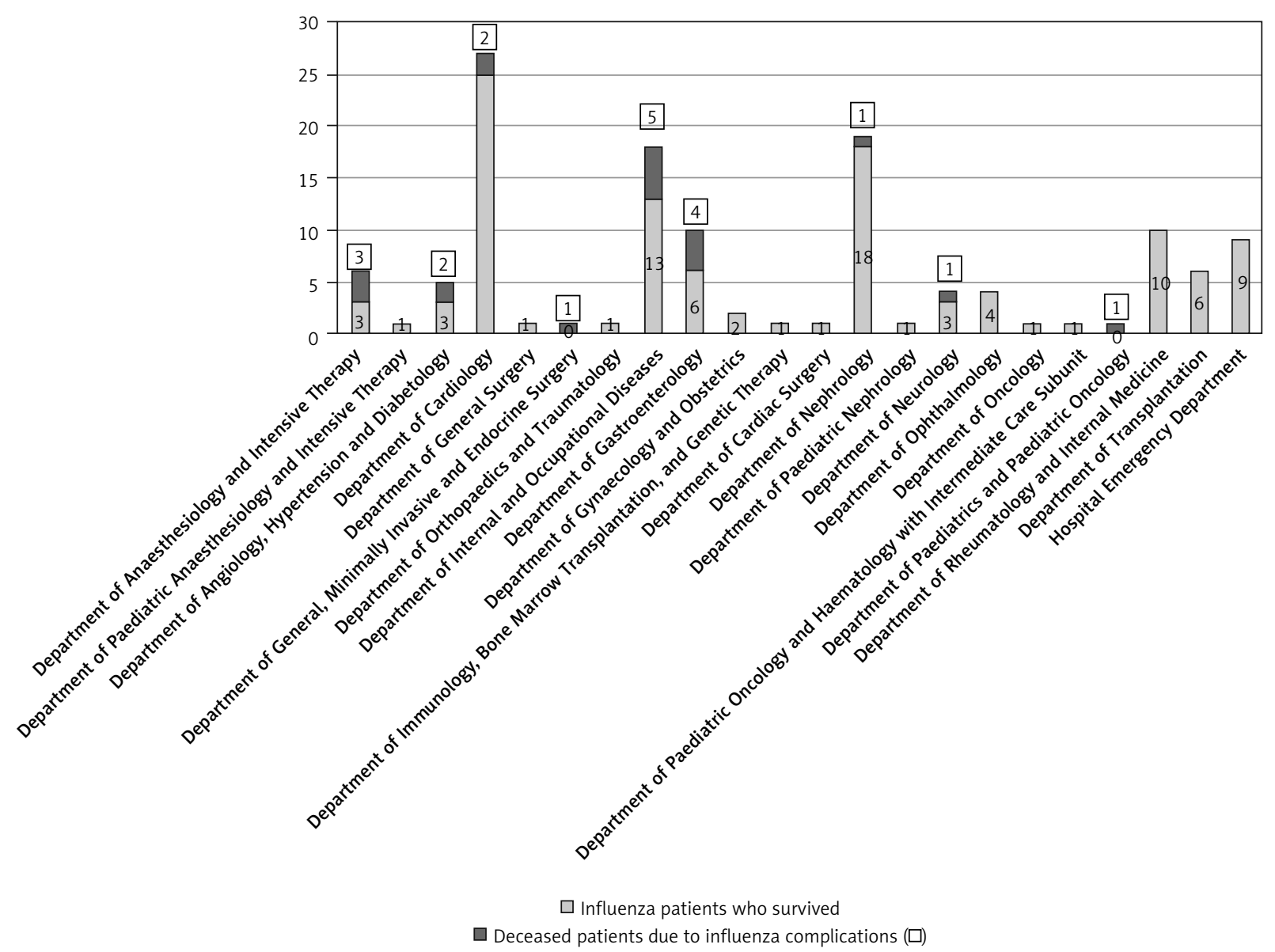

Figure 1. Numbers of patients with confirmed influenza and numbers of deceased patients with confirmed influenza infection, by department 
was determined by rounding up the number of influenza cases to the nearest ten. The random selection was performed using the pseudorandom number generator available in the spreadsheet. The patients' age, length of hospital stay and mortality were analysed. The threshold for statistical significance of differences between the groups was set at $p<0.05$.

\section{Results}

\section{Types of influenza virus}

Among 130 confirmed (by virology testing) influenza cases that occurred in the hospital during the 2018-2019 influenza season in the first quarter of 2019, 103 (77.7\%) cases were caused by the type A influenza virus, subtype A/H1N1/pdm09, while in $27(20.8 \%)$ cases, the type A virus was identified without subtyping. There was no information available on any patients in the study group who contracted influenza despite being vaccinated against influenza in the $2018 / 2019$ season.

Hospital departments where the influenza viral infections were confirmed

Over the study period, influenza cases were confirmed in 22 hospital departments; however, only half of them had more than two cases identified. The influenza infections were mostly observed in the departments related to internal medicine. The largest numbers of influenza cases (84 of total 130) were reported at the Departments of Cardiology (27 cases), Nephrology (19 cases), Internal and Occupational Diseases (18 cases), Gastroenterolo- gy (10 cases) and Rheumatology and Internal Medicine (10 cases) - as presented in Figure 1.

\section{Mortality among patients with confirmed} influenza infection

Deaths of influenza patients were observed in 9 hospital departments. The overall number of fatalities reported in the group of 130 patients with confirmed influenza infection was 20 (15.38\%). The difference in the mortality rates was statistically significant: in the group of patients with influenza infection, the mortality rate was 6.66 -fold greater than in the control group, where it was $2.31 \%$ (6 out of 260 patients).

For the departments related to internal medicine with the largest numbers of influenza cases, a statistically significant relationship was found between incidence of influenza and fatality, and the highest fatality rate among the patients with confirmed influenza infection was reported by the Departments of Gastroenterology (40\%), of Angiology, Hypertension and Diabetology (40\%) and of Internal and Occupational Diseases (27.78\%) - as presented in Table I. Even higher values of this indicator were observed in some departments where fewer patients or even a single patient with confirmed influenza were hospitalized but the statistical significance of the relationship between incidence of influenza and fatality is doubtful due to the very small sample sizes.

\section{Pooled analysis of the parameters under study}

During the study period (first quarter of 2019), statistically significant differences in age, length

Table I. Numbers of patients with confirmed influenza, numbers and percentages of deceased patients with confirmed influenza infection demonstrating statistically significant relationship between influenza incidence rate and fatality rate, by department

\begin{tabular}{|lccc|}
\hline Hospital Department & $\begin{array}{c}\text { Influenza } \\
\text { patients }\end{array}$ & $\begin{array}{c}\text { Patients deceased } \\
\text { due to influenza } \\
\text { complications }\end{array}$ & $\begin{array}{c}\text { \% of deceased } \\
\text { influenza } \\
\text { patients }\end{array}$ \\
\hline Department of Gastroenterology & 10 & 4 & 40.00 \\
\hline Department of Angiology, Hypertension and Diabetology & 5 & 2 & 40.00 \\
\hline Department of Internal and Occupational Diseases & 18 & 5 & 27.78 \\
\hline
\end{tabular}

Table II. Ages and main hospitalization indicators for patients with confirmed influenza infection and for the control group

\begin{tabular}{|c|c|c|c|c|c|c|c|}
\hline \multirow[t]{2}{*}{ Variable } & \multicolumn{3}{|c|}{$\begin{array}{l}\text { Patients with confirmed influen- } \\
\text { za infection }(n=130)\end{array}$} & \multicolumn{3}{|c|}{ Control group $(n=260)$} & \multirow{2}{*}{$\begin{array}{c}\text { Test } \\
\text { result } \\
p \text {-value }\end{array}$} \\
\hline & Mean & Median & $\begin{array}{l}\text { Standard } \\
\text { deviation }\end{array}$ & Mean & Median & $\begin{array}{l}\text { Standard } \\
\text { deviation }\end{array}$ & \\
\hline Age [years] & 61.5 & 66.0 & 20.3 & 49.5 & 59.0 & 25.8 & $<0.05$ \\
\hline $\begin{array}{l}\text { Patient's length of stay } \\
\text { (department) [days] }\end{array}$ & 17.8 & 14.0 & 16.3 & 6.3 & 3.0 & 10.6 & $<0.05$ \\
\hline $\begin{array}{l}\text { Patient's length of stay } \\
\text { (overall hospitalization) [days] }\end{array}$ & 21.3 & 18.0 & 18.3 & 9.9 & 4.0 & 18.6 & $<0.05$ \\
\hline
\end{tabular}


of department stay and overall length of hospital stay were observed between the group of patients with confirmed influenza infection and the control group (Table II).

The pooled analysis of the whole study group and the whole control group showed statistically significant differences in their age (mean: 61.59 and 49.52 years, respectively). However, in the analysis of data from individual departments, it was found that although age-related differences were observed, they were not statistically significant for any hospital department with a number of influenza cases that would be sufficient to determine the statistical significance of the investigated parameters (Figure 2). Thus, it should be assumed that age does not interfere with the findings for the other parameters.

Considering data from the entire hospital, statistically significant differences were found between the study group and the control group for the following parameters: length of stay in the given department and overall length of hospital stay.

The analysis of the individual units showed that among 12 hospital departments where the number of influenza cases was sufficient to per- form an analysis of statistical significance for the parameters under study, statistically significant differences were observed in at least one parameter at the following nine departments: the Department of Anaesthesiology and Intensive Therapy, the Department of Angiology, Hypertension and Diabetology, the Department of Cardiology, the Department of Gastroenterology, the Department of Nephrology, the Department of Neurology, the Department of Ophthalmology, the Department of Transplantation and the Hospital Emergency Department. The only exceptions, where these differences were not confirmed, were the Department of Internal and Occupational Diseases, the Department of Gynaecology and Obstetrics, and the Department of Rheumatology and Internal Medicine.

\section{Length of patients' stay in the hospital departments}

The mean length of hospital stay of those patients with confirmed influenza infection was 21.3 days, which was 2.13-fold longer than that of the control group (10 days).

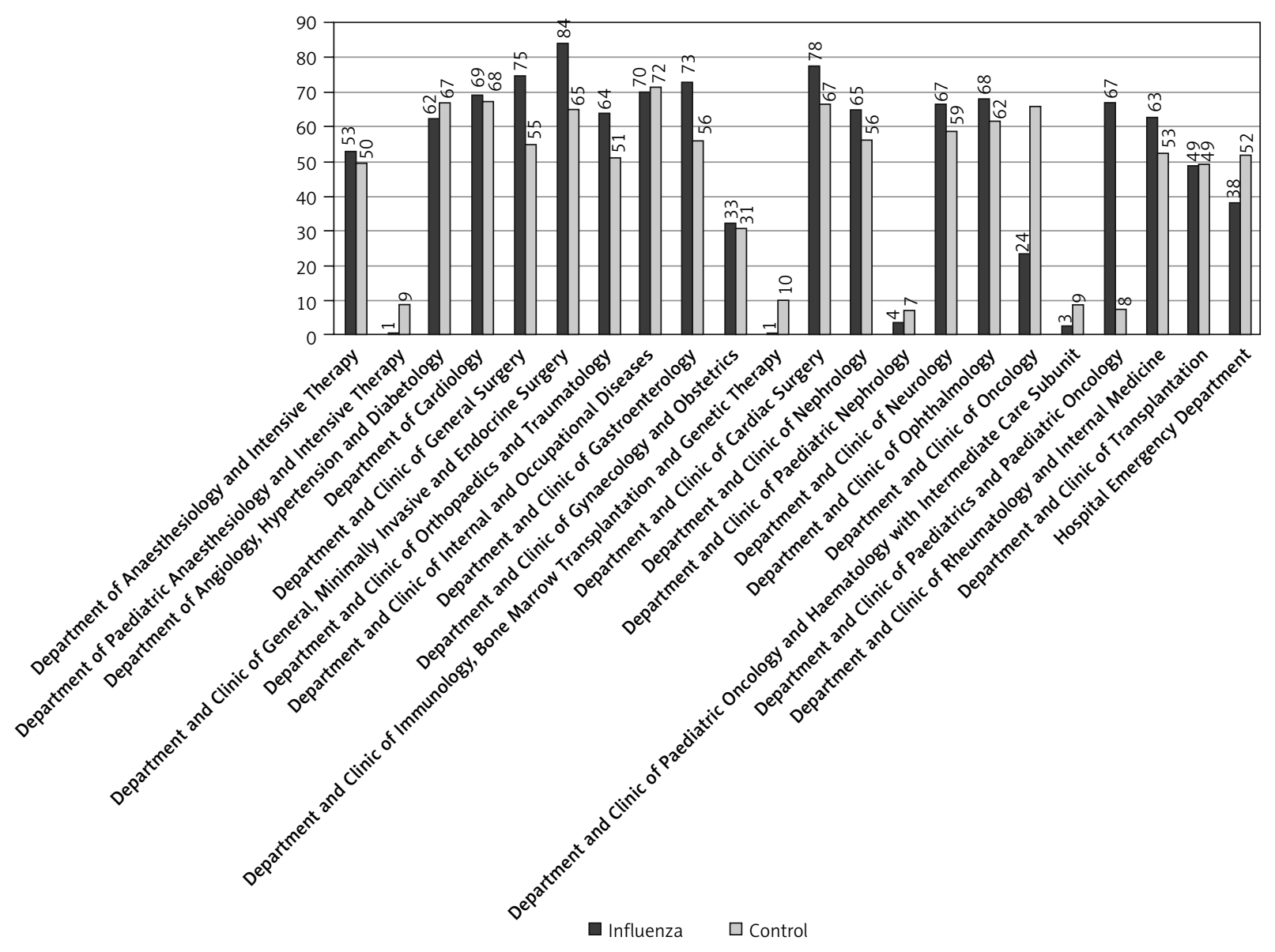

Figure 2. Mean ages of patients with confirmed influenza infection and the control group, by department [years] 
For eight departments, statistically significant differences in the length of stay in a given department were found between the group with confirmed influenza infection and the control group; among the departments with a sufficient number of patients for statistical analysis, such differences were not reported for the Department and Clinic of Internal and Occupational Diseases, the Department and Clinic of Rheumatology and Internal Medicine, or the Hospital Emergency Department (Figure 3).

The largest statistically significant differences in the mean length of stay in a particular hospital department between the patients with confirmed influenza infection and the control group were observed at the Department of Nephrology, the Department of Ophthalmology, the Department of Neurology and the Department of Anaesthesiology and Intensive Therapy: for the patients with influenza, they were 7.08-, 7.08-, 6.57-, and 6.11fold longer than for the control group, respectively. The smallest differences were observed at the Department of Cardiology, the Department of Angiology, Hypertension and Diabetology, the Department of Gastroenterology, and the Department of
Transplantation (2.28-, 3.17-, 3.91- and 4.48-fold longer stay concerning the patients with influenza; Figure 4).

The differences in the length of stay in a given department between the patients with confirmed influenza infection and the control group are even more distinctly illustrated by comparisons of the minimum and maximum lengths of stay and the medians calculated for individual hospital departments. The median length of stay at the Department of Transplantation in the case of the patients with influenza was 16.5 -fold longer than that of the control group. For the Department of Neurology, the minimum length of stay for the patients with confirmed influenza infection was 22fold longer than that of the control group. At the Department of Nephrology, the maximum length of stay for the patients with confirmed influenza infection was 7.4-fold longer than in the control group.

\section{Overall length of hospital stay concerning patients with confirmed influenza infection}

Statistically significant differences in the overall length of patients' hospital stay were also

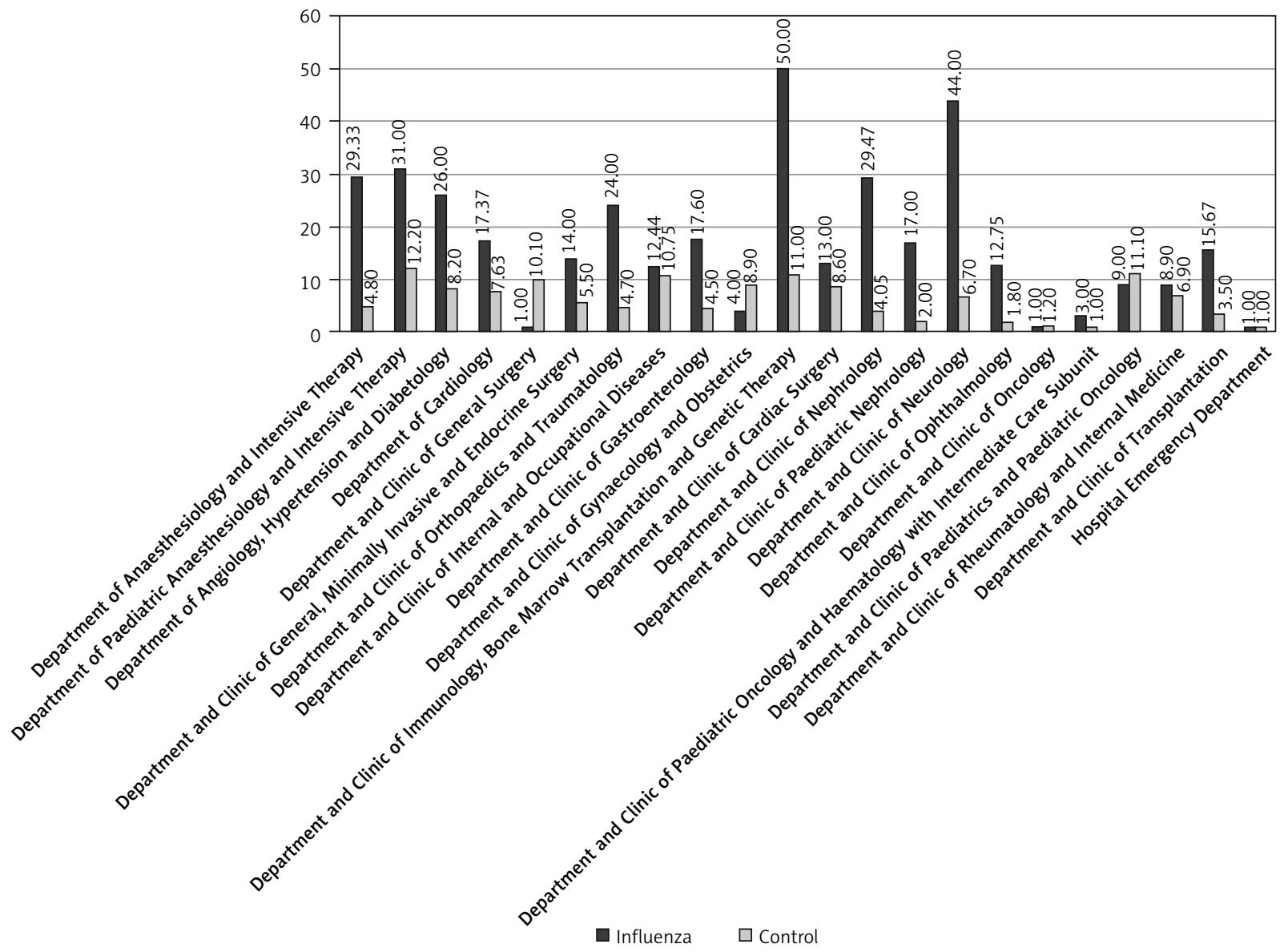

Figure 3. Mean lengths of stay in hospital department for patients with confirmed influenza infection and for the control group [days] 


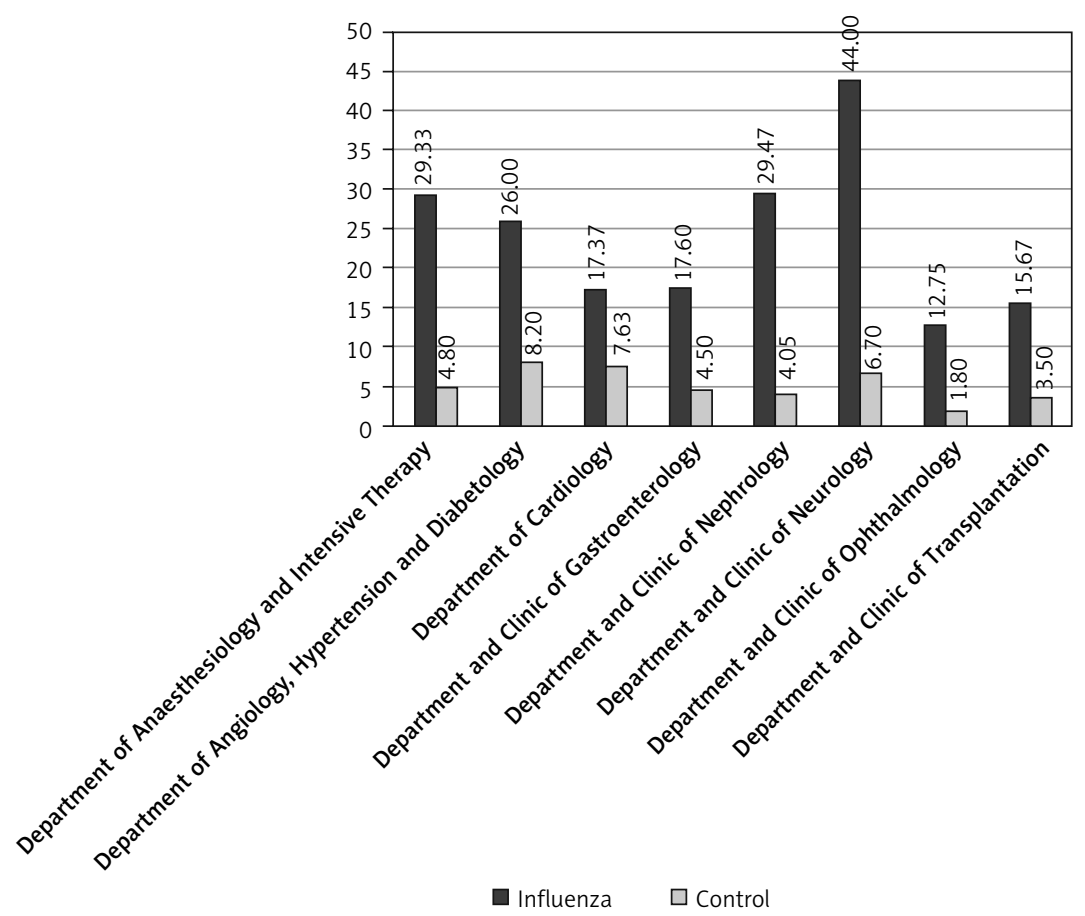

Figure 4. Mean lengths of stay in hospital departments (with statistically significant differences between groups) for patients with confirmed influenza infection and for the control group [days]

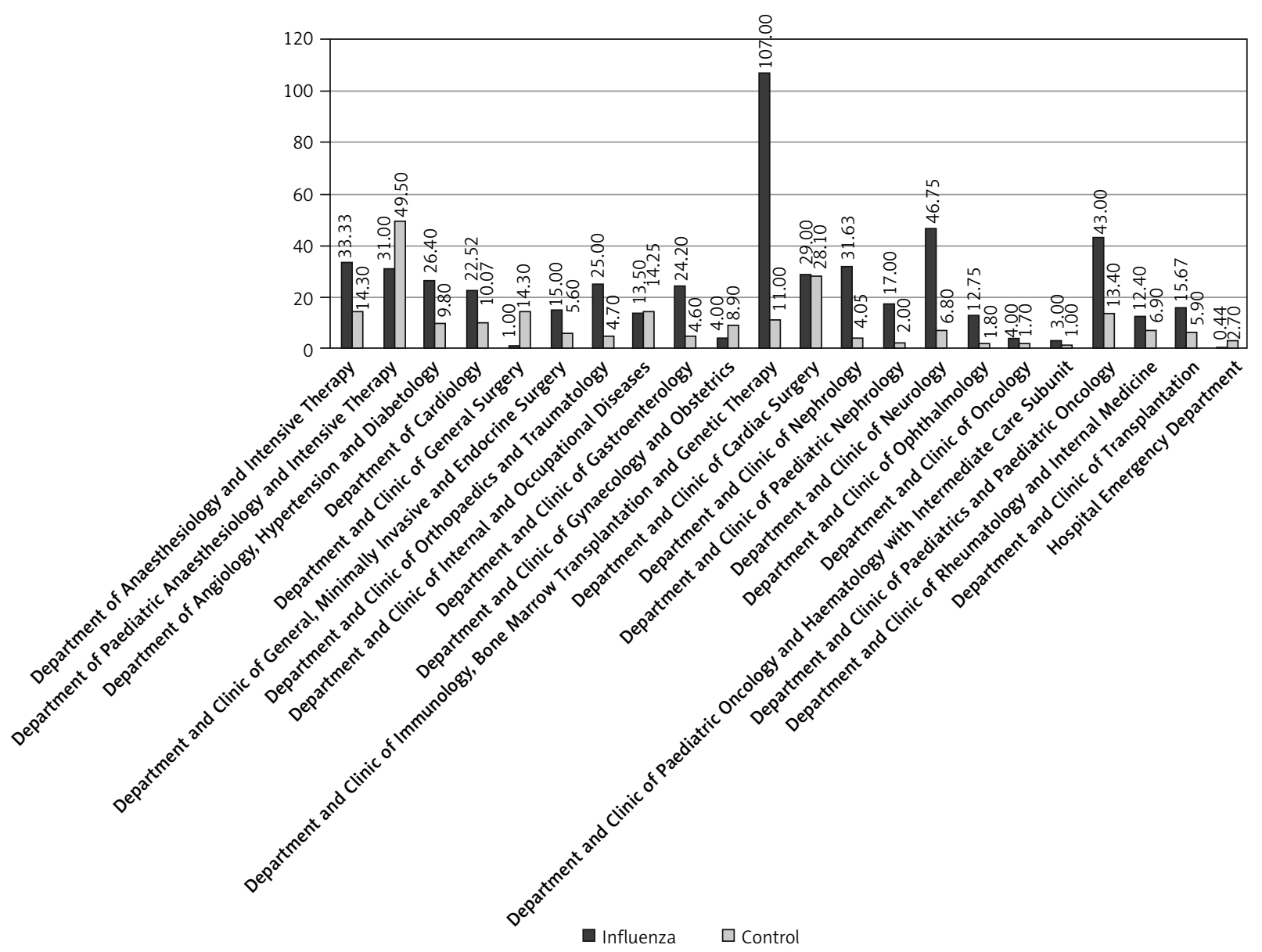

Figure 5. Mean lengths of overall hospital stay for patients with confirmed influenza infection and for the control group [days] 


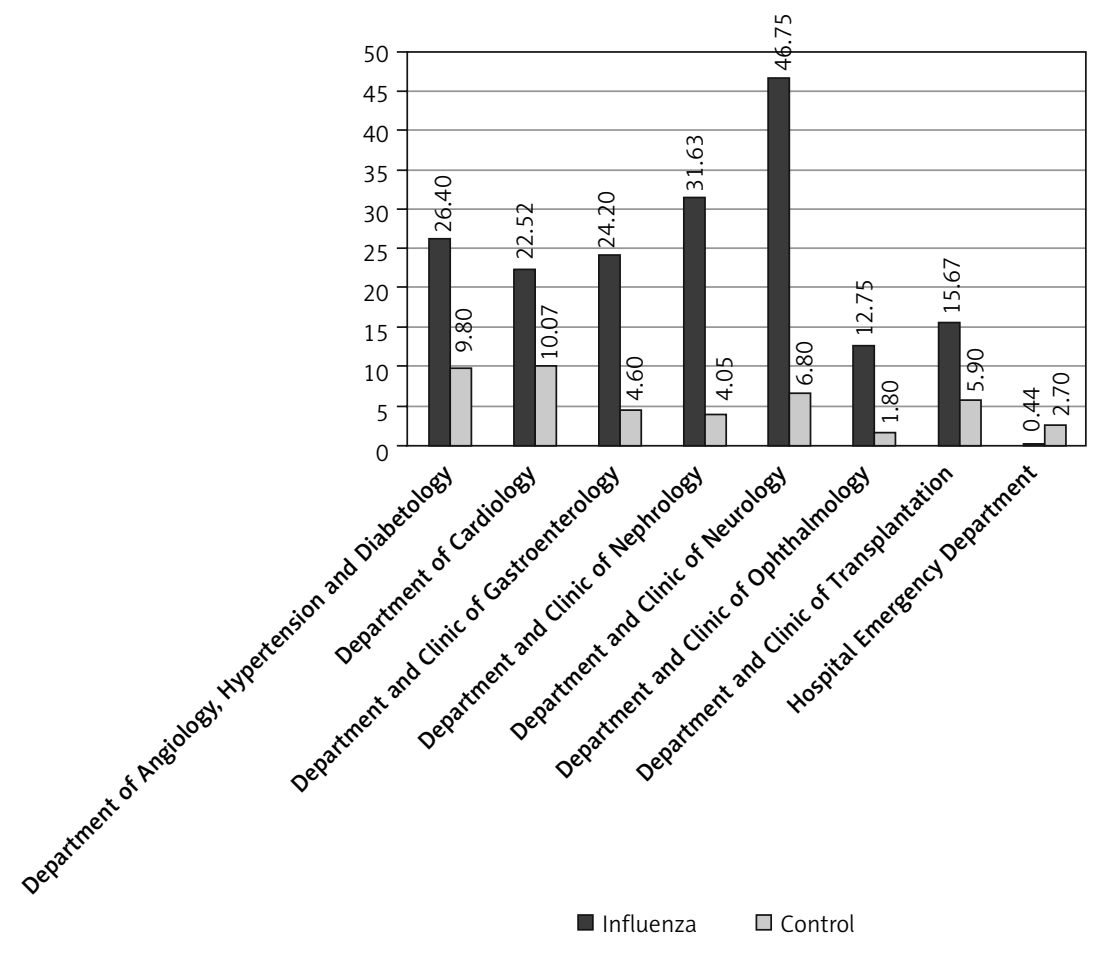

Figure 6. Mean lengths of overall hospital stay for patients with confirmed influenza infection and for the control group (departments with statistically significant differences between groups) [days]

observed in eight departments; among the departments with a sufficient number of patients for statistical analysis, such differences were not reported for the Department of Anaesthesiology and Intensive Therapy, the Department and Clinic of the Internal and Occupational Diseases or the Department of Rheumatology and Internal Medicine (Figure 5).

The largest statistically significant differences in the mean overall length of hospital stay between the patients with confirmed influenza infection and the control group were observed at the Department of Nephrology, the Department of Ophthalmology, the Department of Neurology and the Department of Gastroenterology: for the patients with influenza they were 7.81-, 7.08-, 6.88-, and 5.26-fold longer than in the control group, respectively. The smallest differences were observed at the Department of Cardiology, the Department of Transplantation and the Department of Angiology, Hypertension and Diabetology (2.24-, 2.66- and 2.69-fold longer stay for the patients with confirmed influenza infection). The mean length of stay in the Emergency Department for the patients with influenza was 6.1-fold shorter than that of the control group (Figure 6).

The differences in the overall length of hospital stay between the patients with confirmed influenza infection and the control group are even more distinctly illustrated by comparisons of the minimum and maximum lengths of stay and the medians calculated for the individual hospital departments. In the case of the Department of Oph- thalmology and the Department of Nephrology, the median overall length of stay for the patients with confirmed influenza infection was 13-fold longer than for those in the control group. Concerning the Department of Neurology, the minimum overall length of stay for the patients with confirmed influenza infection was 30-fold longer than for the control group. In the case of the Department of Nephrology, the maximum length of stay for the patients with confirmed influenza infection was 7.4-fold longer than that of the control group.

\section{Discussion}

In Poland, during the 2018-2019 influenza season (1 September 2018 to 22 July 2019), 4,491,879 cases of influenza or influenza-like infection were reported, which is approximately $15 \%$ less than during the previous influenza season. There were 17,118 referrals to hospital for patients with suspected influenza, which is a $6 \%$ reduction compared to the previous influenza season. However, over the entire epidemic period, as many as 150 fatal cases due to influenza complications were reported, which was 100 more cases than during the previous influenza season; the deaths were mainly observed among patients aged 65 and older. The A/H1N1/pdm09 virus strain was responsible for $77.5 \%$ of the influenza cases, the $A$ / H3N2 strain was the cause of $4.3 \%$, type A influenza virus without subtyping caused $17.1 \%$, and type B caused $1.1 \%$ [21]. The data from the University Hospital in Wroclaw analysed in this study 
revealed the same proportion of the $\mathrm{A} / \mathrm{H} 1 \mathrm{~N} 1 /$ pdm09 strain as for the general Polish population, though slightly more cases were caused by influenza type A viruses without subtyping and fewer infections were due to type $B$ viruses.

According to the State Sanitary Inspector for the Lower Silesian Province (non-published data), 119,641 overall cases of influenza and suspected influenza, 447 laboratory-confirmed influenza cases and 330 influenza-related hospitalizations were reported for the entire Lower Silesian Province in the first quarter of 2019 during the influenza period, while for the city of Wroclaw, the numbers were 286 laboratory-confirmed cases and 164 hospitalizations, respectively. It should be noted in this context that within the Lower Silesian Province, the laboratory-confirmed influenza cases were reported overall by 31 inpatient care institutions but as many as 130 infections were confirmed at the University Hospital in Wroclaw alone, constituting $45.5 \%$ of the laboratory-confirmed influenza infections and $78.3 \%$ of the influenza-related hospitalizations in the entire city of Wroclaw, constituting $27.3 \%$ of the overall laboratory-confirmed influenza infections for the whole Province of Lower Silesia and $39.4 \%$ of the influenza-related hospitalizations. These figures may suggest a significant underestimation of the influenza cases reported in the Lower Silesian Province. It is consistent with the reports from other countries $[8,9]$. In the case of Poland, this may result from the limited actual availability of laboratory influenza tests faced by healthcare institutions that, unlike the University Hospital, do not have their own microbiology laboratories with proper testing potential, as well as from the small number of the cases being reported that meet the clinical diagnostic criteria for influenza, leading to diagnoses of other diseases instead, which is consistent with relevant source data in the literature.

According to the literature, the largest numbers of influenza-related cases and fatalities should be expected in more susceptible populations: among young children, the elderly, chronically ill patients suffering from cardiovascular or respiratory disease and immunosuppressed patients [14, 19]. This has been confirmed by data from the University Hospital in Wroclaw, where the largest numbers of influenza cases were reported for the departments related to internal medicine, in particular, the Department of Cardiology, the Department of Nephrology with dialysis therapy, and the Department of Internal and Occupational Diseases (which also admits patients with chronic lung diseases; Figure 1).

Moreover, source data suggest that influenza infections increase the risk of death $[8,10]$, which was reflected in the data from the University Hos- pital in Wroclaw: the risk in the group of patients with confirmed influenza infection was as high as $15.38 \%$ compared to only $2.31 \%$ in the control group. In the departments related to internal medicine where patients with chronic diseases are hospitalized, the risk of death among the patients with confirmed influenza infection was markedly higher, ranging from $27.78 \%$ in the Department of Internal and Occupational Diseases to as high as $40 \%$ at the Departments of Angiology, Hypertension, and Diabetology and of Gastroenterology (Table I).

According to the literature data, influenza infections not only increase the risk of hospitalization, but they also prolong the hospital stay [10, 11]. This has been confirmed by data from the University Hospital in Wroclaw, as statistically significant differences were found between the patients with confirmed influenza infection and the control group in both the length of patients' stay in a given department and the overall length of hospital stay (Table II). Compared to the control group, the mean hospitalization period was over twice as long among the patients with confirmed influenza infection.

Not all departments of the University Hospital in Wroclaw had enough hospitalized influenza patients to demonstrate statistically significant differences in comparison with the control group at the significance level chosen for the study. In most departments where meaningful analysis was possible, statistically significant differences in at least one of the parameters being studied were found between the two groups; the exceptions were the Departments of Internal and Occupational Diseases, of Rheumatology and Internal Medicine, and of Gynaecology and Obstetrics. The lack of such differences is particularly surprising in the latter case as pregnant women - especially during the last trimester - are at higher risk of a severe disease course and serious complications [22].

Considering the University Hospital departments where statistically significant differences in the length of patients' stay in a particular department were observed, the mean length of stay of patients with confirmed influenza infection was remarkably longer (2.28- to 7.08-fold) than that of the patients in the control group (Figure 4), which is consistent with the conclusions in the source literature [10, 11, 23]. Even more extreme differences (ten-fold or higher), which are unfavourable for patients with confirmed influenza infection, were found in the comparisons between the median, minimum, and maximum lengths of stay of the two groups.

Also, the analysis of the overall length of hospital stay in the departments with statistically significant differences in this indicator revealed 
that the mean overall hospitalization period for the patients with confirmed influenza infection was markedly longer (2.28- to 7.81-fold) than for the control group, which is consistent with reports in the literature $[10,11]$. One exception was the Emergency Department, where the length of stay for patients with influenza infection/confirmed influenza was 6.1-fold shorter than for the control group (Figure 6). This should be attributed to these patients' quick diagnosis and discharge from the Emergency Department with instructions, with no need for hospitalization for influenza and its complications or with contraindications to a scheduled hospital admission for other reasons. Even more extreme differences (over several dozen-fold), which are unfavourable to patients with confirmed influenza infection, were found in the comparisons of the median, minimum, and maximum lengths of the overall hospital stay between the two groups.

The burdens and costs of influenza can be effectively mitigated not only by enforcing responsible social behaviours preventing spread of the infection in the general population but also by effectively ensuring massive participation in vaccination programmes [23].

In conclusion, during the 2018-2019 influenza season, the types of influenza viruses identified in the patients of the University Hospital in Wroclaw were notably comparable to those occurring in the general population in Poland. However, the disproportionately high share of the number of laboratory-confirmed influenza infections and influenza-related hospitalizations reported by the University Hospital, in the respective total numbers reported in the whole Lower Silesian Province, suggests a significant underestimation of the influenza cases in the province that may result from incomplete influenza reporting by healthcare institutions or limited availability of laboratory influenza tests.

The largest numbers of influenza cases and the highest risk of death were observed in the hospital departments related to internal medicine where patients with cardiac, lung, and renal diseases (and frequently many comorbidities) or elderly patients were hospitalized. Also in general, the risk of death in the group of patients with confirmed influenza was over six-fold higher on average than among the other patients. For the entire hospital, the mean length of hospital stay was 2.13-fold longer among the patients with confirmed influenza than in the control group.

Both the increased risk of death and prolonged hospital stays of patients with influenza infection cause significant social costs. Moreover, in the case of patients suffering from influenza, prolonged hospital stays alone translate directly into a significant increase in the inpatient treat- ment costs that should be adequately mirrored in elevated level of financing by medical insurance institutions.

Considering the above, it is necessary to take action in order to decrease both the social burden and the hospital treatment costs resulting from seasonal influenza infections. It requires public health actions, primarily popularizing influenza vaccines and promoting responsible attitudes in society in cases of influenza-like symptoms, which would allow patients with suspected influenza to be isolated early on and provided with proper outpatient medical care that may prevent many excessive hospitalizations and deaths.

There are significant clinical and epidemiological similarities between influenza and another, recently emerged, infectious disease of the respiratory tract, i.e. COVID-19. It is justified to assume that coronavirus SARS-CoV-2 infections are associated with a significant social burden and increased hospital treatment costs that are underestimated similarly as in the case of influenza virus infections. These burdens and costs of COVID-19 can be mitigated in similar ways as in the case of seasonal influenza - not only by enforcing responsible social behaviours preventing spread of the infection in the general population but also by effectively ensuring massive participation in vaccination programmes.

\section{Conflict of interest}

\section{????}

\section{References}

1. Bilcke J, Coenen S, Beutels P. Influenza-like-illness and clinically diagnosed flu: Disease burden, costs and quality of life for patients seeking ambulatory care or no professional care at all. PLoS One 2014; 7: e102634.

2. Behzadi M, Ziyaeyan M, Alborzi A. A diagnostic one-step real-time reverse transcription polymerase chain reaction method for accurate detection of influenza virus type A. Arch Med Sci 2016; 12: 1286-92.

3. Wrotek A, Czajkowska M, Zawlocka E, Jackowska T. Socioeconomic costs of influenza complications in hospitalized children. Arch Med Sci . DOI: https://doi. org/10.5114/aoms.2020.99060.

4. Brydak L, Masny A. Wytyczne do przygotowania placówek medycznych na wypadek wystąpienia pandemii grypy. NIZP-PZH, Warszawa 2018. Source: https://gis. gov.pl/wp-content/uploads/2018/07/Wytyczne-doplanu-pandemicznego-grypy-NIZP-PZH.pdf [accessed 30.10.2019].

5. Tempia S, Moyes J, Cohen A, et al. Health and economic burden of influenza-associated illness in South Africa, 2013-2015. Influenza Other Respir Viruses 2019; 13: 484-95.

6. Brydak L. Grypa, pandemia grypy mit czy realne zagrożenie? Wydawnictwo RYTM, Warsaw 2008; 1-492.

7. Chlebicki A, Kozioł M. Homogeneous Group approach to Elixhauser comorbidity for hospital death using administrative data. Arch Med Sci 2020; 16: 321-36. 
8. Sprenger M, Mulder P, Beyer W, Van Strik R, Masurel N. Impact of influenza on mortality in relation to age and underlying disease, 1967-1989. Int J Epidemiol 1993; 22: 334-40.

9. Aschwanden C. Debunking the false claim that COVID death counts are inflated. Scientific American 20.10.2020. Source: https://www.scientificamerican. com/article/debunking-the-false-claim-that-coviddeath-counts-are-inflated/ [accessed: 24.11.2020].

10. Rothberg M, Haessler S, Brown R. Complications of viral influenza. Am J Med 2008; 121: 258-64.

11. Kovács G, Kaló Z, Jahnz-Rozyk K, et al. Medical and economic burden of influenza in the elderly population in central and eastern European countries. Human Vaccines Immunother 2014; 10: 428-40.

12. Knapik P, Knapik M, Trejnowska E, et al. Should we admit more patients not requiring invasive ventilation to reduce excess mortality in Polish intensive care units? Data from the Silesian ICU Registry. Arch Med Sci 2019; 15: 1313-20.

13. Ochman M, Urlik M, Tatoj Z, et al. Retrospective cohort study of patients qualified for lung transplantation due to idiopathic pulmonary fibrosis - single-centre experience. Arch Med Sci 2020; 16: 621-6.

14. Bartoszko J, Loeb M. The burden of influenza in older adults: meeting the challenge. Aging Clin Exp Res 2021; 33: 711-7.

15. Lin Y, Li T, Chen W. Ischaemic stroke and influenza A H1N1 vaccination: a case report. Arch Med Sci 2011; 7: 345-8.

16. Kim T, Johnstone J, Loeb M. Vaccine herd effect. Scand J Infect Dis 2011; 43: 683-9.

17. Paplicki M, Susło R, Najjar N, Ciesielski P, Augustyn J, Drobnik J. Conflict of individual freedom and community health safety: legal conditions on mandatory vaccinations and changes in the judicial approach in the case of avoidance. Fam Med Prim Care Rev 2018; 20: 389-95:

18. Muras M, Krajewski J, Nocun M, Godycki-Cwirko M. A survey of patient behaviours and beliefs regarding antibiotic self-medication for respiratory tract infections in Poland. Arch Med Sci 2013; 9: 854-7.

19. Patriarca P, Weber J, Parker R, et al. Efficacy of influenza vaccine in nursing homes: reduction in illness and complications during an influenza A (H3N2) epidemic. JAMA 1985; 253: 1136-9.

20. Pavličević I, Škrabić S. Malički M, Merćep A, Marušić M, Marušić A. Decisional conflict and vaccine uptake: cross-sectional study of 2012/2013 influenza season in Croatia. Arch Med Sci 2015; 11: 788-95.

21. Brydak L. Jak wiele zachorowań na grypę występuje w Polsce? PZH-NIZP. Szczepienia Info. Source: http:// szczepienia.pzh.gov.pl/szczepionki/grypa/3/\#jak-wielezachorowan-na-grype-wystepuje-w-polsce [accessed 30.10.2019].

22. Szenborn L, Matkowska-Kocjan A. Grypa u ciężarnych - leczenie i profilaktyka. Medycyna po Dyplomie 2010; 19: 98-103.

23. Paplicki M, Susto R, Benedikt A, Drobnik J. Effectively enforcing mandatory vaccination in Poland and worldwide. Fam Med Prim Care Rev 2020; 22: 252-6. 